\title{
Increased DNA Dicarbonyl Glycation and Oxidation Markers in Patients with Type 2 Diabetes and Link to Diabetic Nephropathy
}

\author{
Sahar Waris, ${ }^{1}$ Brigitte M. Winklhofer-Roob, ${ }^{2}$ Johannes M. Roob, ${ }^{3}$ Sebastian Fuchs, ${ }^{2}$ \\ Harald Sourij, ${ }^{4}$ Naila Rabbani, ${ }^{1,5}$ and Paul J. Thornalley ${ }^{1,5}$ \\ ${ }^{1}$ Warwick Medical School, Clinical Sciences Research Laboratories, University of Warwick, University Hospital, \\ Coventry CV2 2DX, UK \\ ${ }^{2}$ Human Nutrition \& Metabolism Research and Training Center Graz, Institute of Molecular Biosciences, \\ Karl Franzens University, 8010 Graz, Austria \\ ${ }^{3}$ Clinical Division of Nephrology, Department of Internal Medicine, Medical University of Graz, 8036 Graz, Austria \\ ${ }^{4}$ Clinical Division of Endocrinology and Nuclear Medicine, Department of Internal Medicine, Medical University of Graz, \\ 8036 Graz, Austria \\ ${ }^{5}$ Warwick Systems Biology Centre, Senate House, University of Warwick, Coventry CV4 7AL, UK
}

Correspondence should be addressed to Naila Rabbani; n.rabbani@warwick.ac.uk

Received 1 January 2015; Revised 14 March 2015; Accepted 23 March 2015

Academic Editor: Patrizio Tatti

Copyright (C) 2015 Sahar Waris et al. This is an open access article distributed under the Creative Commons Attribution License, which permits unrestricted use, distribution, and reproduction in any medium, provided the original work is properly cited.

\begin{abstract}
Aim. The aim of this study was to assess the changes of markers of DNA damage by glycation and oxidation in patients with type 2 diabetes and the association with diabetic nephropathy. Methodology. DNA oxidation and glycation adducts were analysed in plasma and urine by stable isotopic dilution analysis liquid chromatography-tandem mass spectrometry. DNA markers analysed were as follows: the oxidation adduct 7,8-dihydro-8-oxo-2'-deoxyguanosine (8-OxodG) and glycation adducts of glyoxal and methylglyoxal-imidazopurinones GdG, MGdG, and $\mathrm{N}_{2}-(1, R / S$-carboxyethyl)deoxyguanosine (CEdG). Results. Plasma 8-OxodG and GdG were increased 2-fold and 6-fold, respectively, in patients with type 2 diabetes, with respect to healthy volunteers. Median urinary excretion rates of 8-OxodG, GdG, MGdG, and CEdG were increased 28-fold, 10-fold, 2-fold, and 2-fold, respectively, in patients with type 2 diabetes with respect to healthy controls. In patients with type 2 diabetes, nephropathy was associated with increased plasma 8-OxodG and increased urinary GdG and CEdG. In a multiple logistic regression model for diabetic nephropathy, diabetic nephropathy was linked to systolic blood pressure and urinary CEdG. Conclusion. DNA oxidative and glycation damagederived nucleoside adducts are increased in plasma and urine of patients with type 2 diabetes and further increased in patients with diabetic nephropathy.
\end{abstract}

\section{Introduction}

Reactive oxygen species (ROS) and dicarbonyl metabolites, glyoxal and methylglyoxal, are some of the most reactive metabolites present in human metabolism. They are increased in diabetes and diabetic nephropathy [1-3]. ROS react with deoxyguanosine residues of DNA to form 8-dihydro-8oxo- $2^{\prime}$-deoxyguanosine (8-OxodG). The DNA content of 8 -OxodG residues was increased in lymphocyte DNA in clinical diabetes [4]. Reactive dicarbonyl intermediates of endogenous glycation, glyoxal and methylglyoxal, also react with deoxyguanosine residues of DNA to form mainly imidazopurinone adducts. Glyoxal forms 3-(2'-deoxyribosyl)-6, 7-dihydro-6, 7-dihydroxyimidazo-[2,3-b] purin9(8)one (GdG) and methylglyoxal forms two imidazopurinone structural isomers, 3-(2'-deoxyribosyl)-6,7-dihydro6, 7-dihydroxy-6/7-methylimidazo-[2,3-b]purine-9(8)one (MGdG). DNA glycation by methylglyoxal also forms two stereoisomers, $\mathrm{N}_{2}$-modified derivatives, $\mathrm{N}_{2}-(1, R / S$-carboxyethyl)-deoxyguanosine (CEdG) [5], Figure 1(a). GdG, MGdG, and CEdG are nucleotide advanced glycation endproducts (AGEs). Methylglyoxal-derived MGdG was a quantitatively major adduct of endogenous damage in healthy volunteers [5]. Damage to DNA by endogenous glyoxal and 


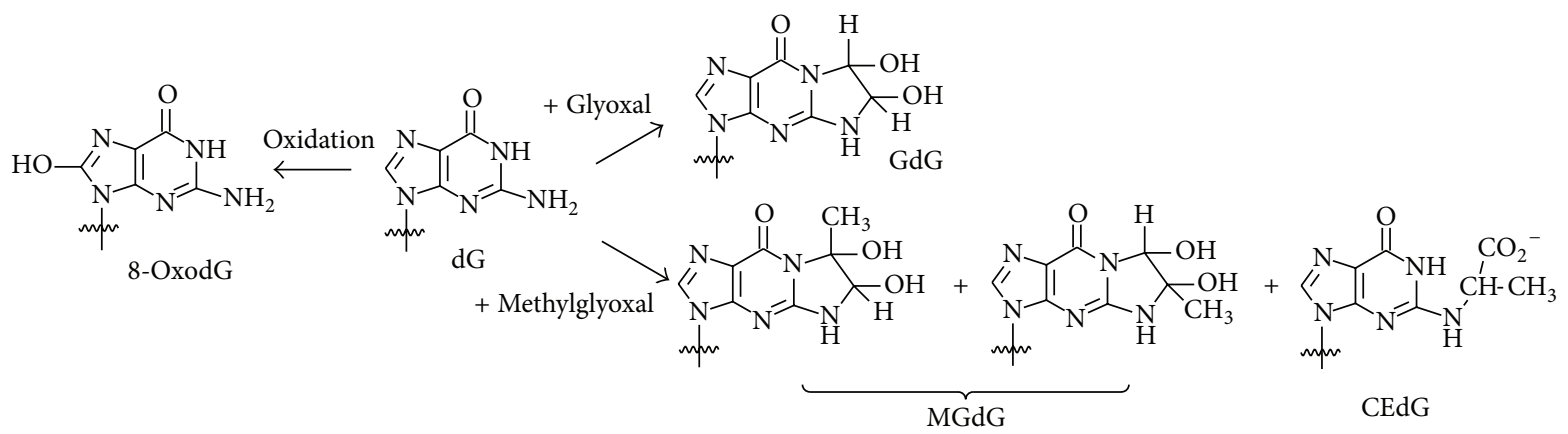

(a)

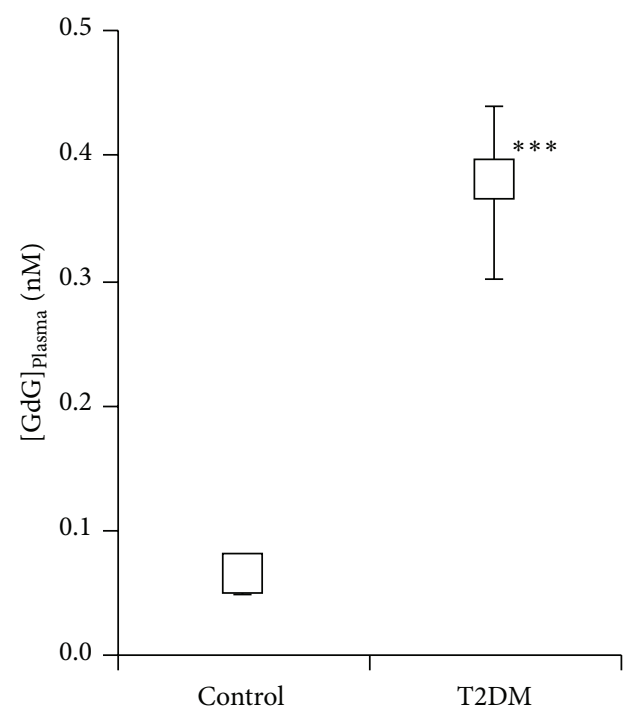

(b)

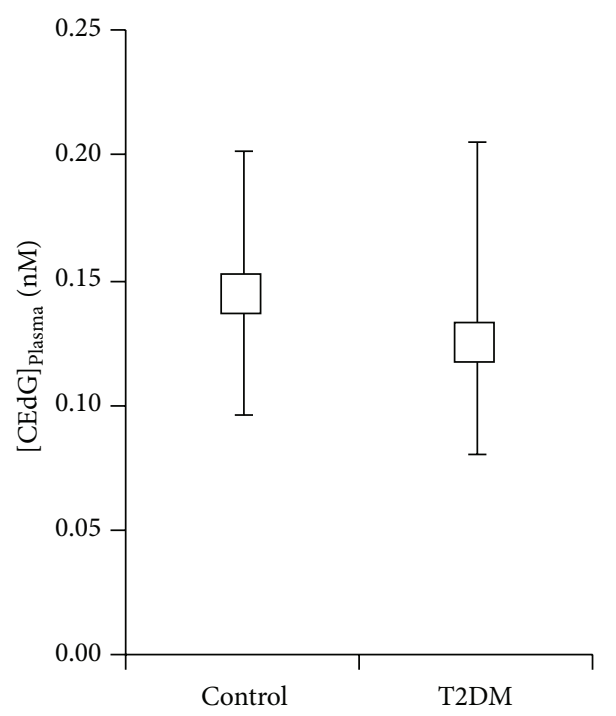

(d)

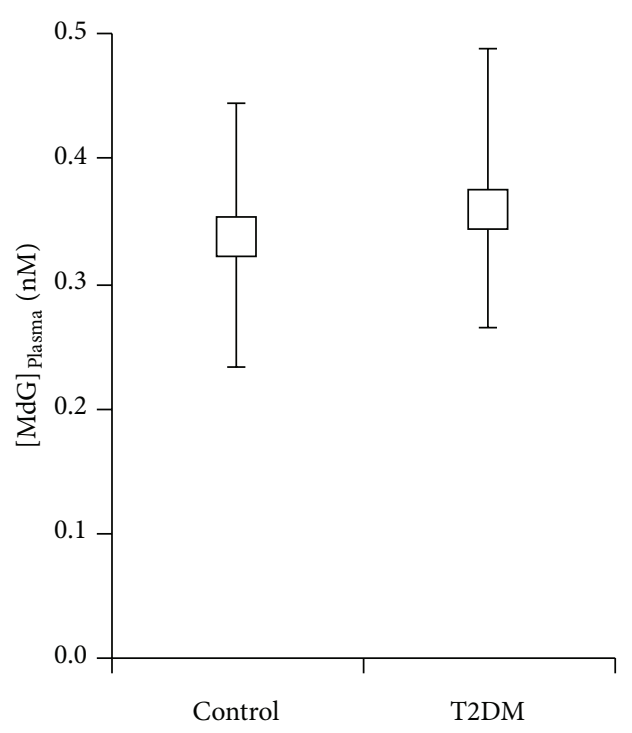

(c)

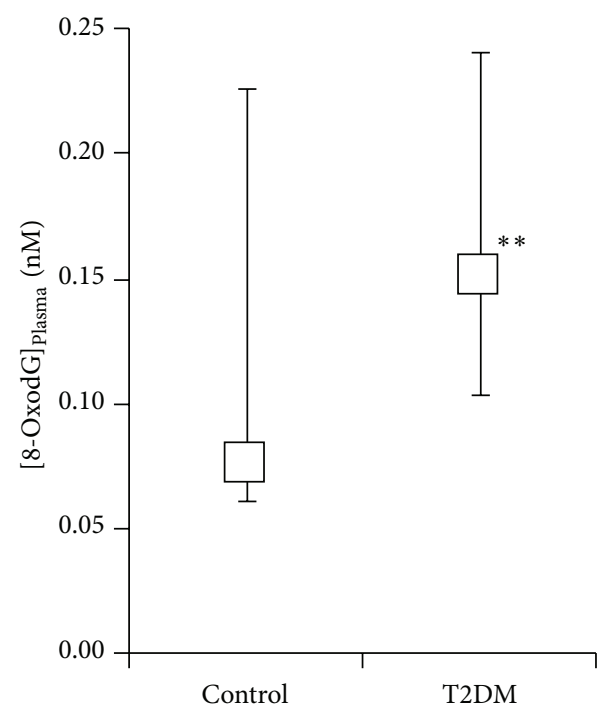

(e)

FIGURe 1: Continued. 


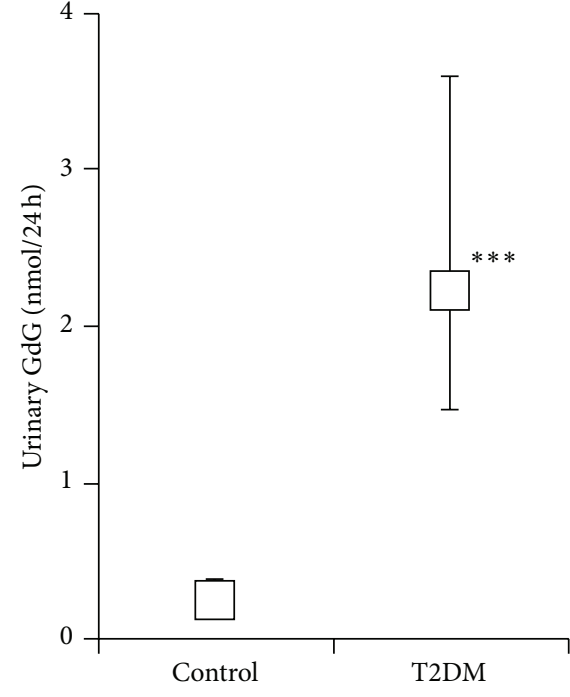

(f)

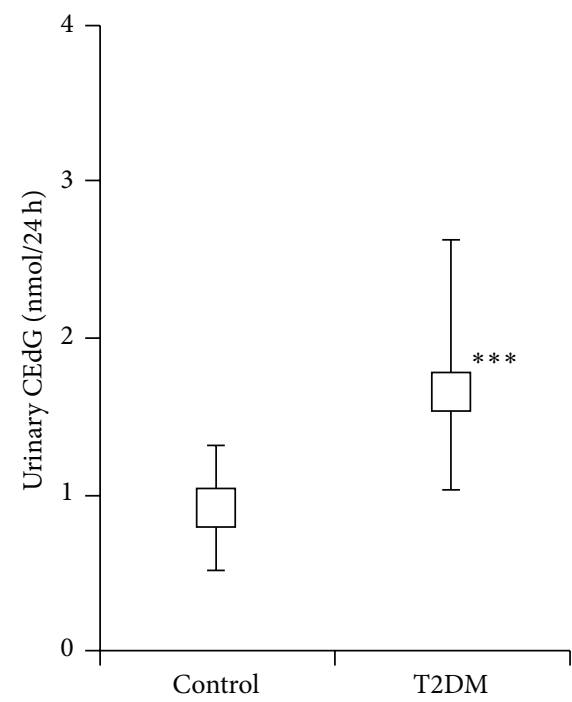

(h)

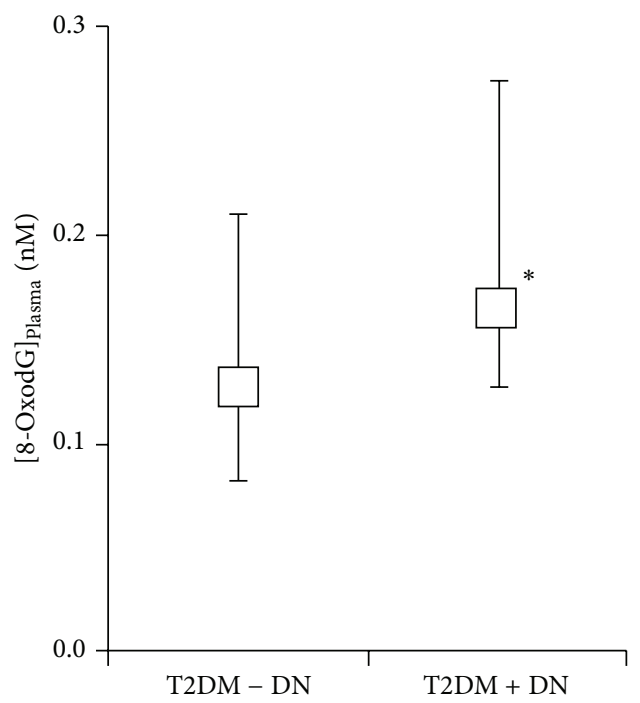

(j)

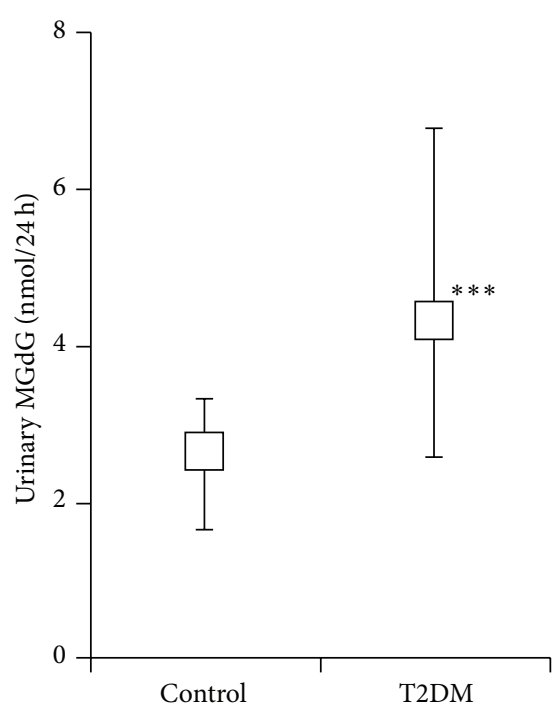

(g)

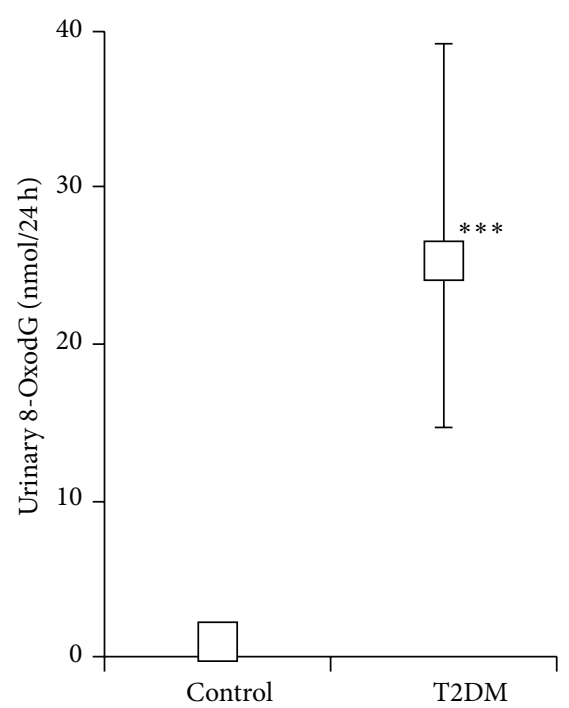

(i)

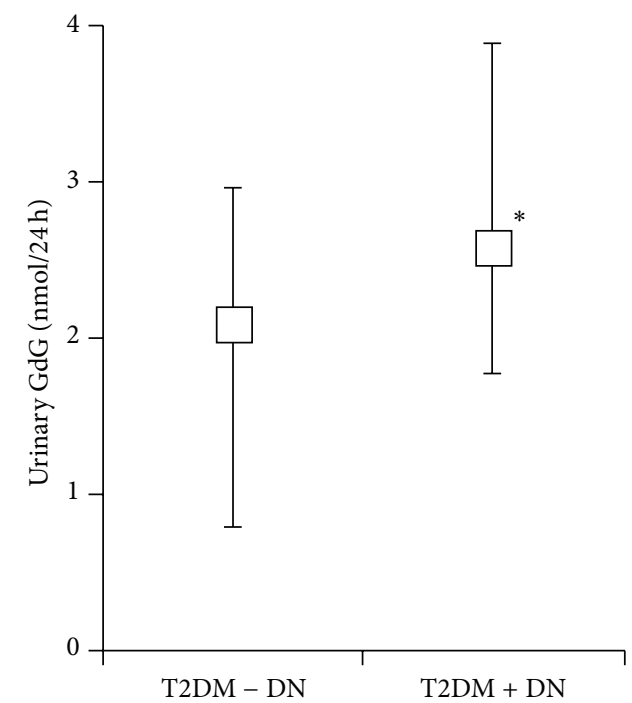

(k)

FIgURE 1: Continued. 


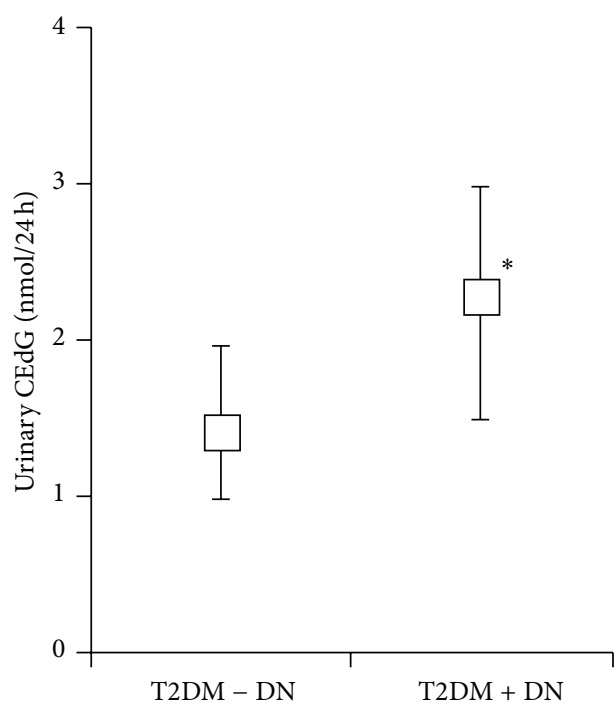

(1)

FIGURE 1: (a) Formation of glycation and oxidation adducts of deoxyguanosine. The common $2^{\prime}$-deoxyribosyl group has been omitted for clarity. (b)-(i) Nucleotide glycation and oxidation adducts in plasma and urine of healthy controls subjects and patients with type 2 diabetes. Plasma: (b) GdG, (c) MGdG, (d) CEdG, and (e) 8-OxodG. Urine: (f) GdG, (g) MGdG, (h) CEdG, and (i) 8-OxodG. (j)-(l) Nucleotide glycation and oxidation adducts in plasma and urine in patients with type 2 diabetes with and without nephropathy. (j) Plasma 8-OxodG, (k) urinary GdG, and (l) urinary CE-dG. Data are median with the upper and lower quartile as error bars. Significance: ${ }^{*} P<0.05,{ }^{* *} P<0.01$, and ${ }^{* * *} P<0.001$; Mann-Whitney $U$.

methylglyoxal is suppressed by glyoxalase 1 (Glo1) of the cytoplasmic glyoxalase system [6]. The expression and activity of Glol in the kidney were decreased in experimental models of diabetic nephropathy-streptozotocin-induced diabetes in mice and rats and the $\mathrm{db} / \mathrm{db}$ diabetic mouse [7-9]. This is associated with increased dicarbonyl glycation of proteins linked to the development of diabetic nephropathy [10]. Increased urinary 8-OxodG excretion has been found in patients with diabetes type 2 diabetes (T2DM), with further increases associated with the presence of microvascular complications, and was a predictor of diabetic nephropathy $[11,12]$.

Nucleotide AGEs are associated with DNA single-strand breaks and increased mutation frequencies [13]. Oxidised and glycated nucleosides are removed from DNA by nucleotide excision repair of DNA damaged by oxidation and glycation. Recent functional genomic studies of Glol have linked dicarbonyl glycation to the development of diabetic nephropathy $[14,15]$. The plasma concentration and urinary fluxes of glyoxal and methylglyoxal-derived nucleoside AGEs in diabetes and diabetic nephropathy are unknown.

Reliable quantitation of oxidised and glycated nucleosides has proven difficult because of inadequate sensitivity and specificity of detection responses and poor adduct stability and recovery during preanalytic processing. Initial studies involved ${ }^{32}$ P-labelling studies of DNA digests [16] and immunoassay [17]. Stable isotopic dilution analysis liquid chromatography with tandem mass spectrometric detection (LC-MS/MS) has been used for assay of 8-OxodG [18]. For estimation of both DNA glycation and oxidation adducts, the high specificity and sensitivity of LC-MS/MS makes this the preferred method for DNA damage marker analysis.
We developed a stable isotopic dilution analysis LC-MS/MS method to concurrently quantify GdG, MGdG, CEdG, and 8OxodG in plasma and urinary ultrafiltrate [5]. 8-OxodG has been studied extensively in plasma and urine as a biomarker of oxidative damage [18] whereas the diagnostic utility of DNA glycation adducts is unknown.

Herein we analysed DNA oxidation and glycation markers in plasma and urine of patients with T2DM with and without diabetic nephropathy. Healthy volunteers served as controls. The outcome revealed marked increases in DNA damage in T2DM and further increase of selected markers in diabetic nephropathy. Increased urinary excretion of glycation adducts GdG and CEdG was indicative of diabetic nephropathy.

\section{Methods}

2.1. Participants. Patients with type 2 diabetes and overt nephropathy (T2DM + DN) and patients with T2DM and without overt nephropathy (T2DM - DN) were recruited in the EU-funded PREDICTIONS project [19]. Healthy volunteers were recruited in the EU-funded VITAGE project [20]. Both studies were conducted at the Human Nutrition and Metabolism Research and Training Center, Karl Franzens University of Graz, Austria, and the Clinical Division of Nephrology and the Clinical Division of Endocrinology and Nuclear Medicine, Department of Internal Medicine, Medical University of Graz, Austria. The study protocols were approved by the Ethics Committee of the Medical University of Graz, Austria, and written informed consent was obtained from all study subjects. Diagnosis of diabetes was established in accordance with the WHO criteria: 
TABLE 1: Characteristics of human subjects recruited for this study.

\begin{tabular}{|c|c|c|c|}
\hline Subject group & Control & T2DM-DN & $\mathrm{T} 2 \mathrm{DM}+\mathrm{DN}$ \\
\hline$n$ & 28 & 28 & 28 \\
\hline Age (years) & $61 \pm 8$ & $63 \pm 6$ & $60 \pm 10$ \\
\hline Gender $(\mathrm{M} / \mathrm{F})$ & $28 / 0$ & $20 / 8$ & $20 / 8$ \\
\hline BMI $\left(\mathrm{kg} / \mathrm{m}^{2}\right)$ & $26.1 \pm 1.8$ & $28.5 \pm 5.0^{*}$ & $33.2 \pm 5.0^{* * *, \mathrm{OOO}}$ \\
\hline Fasting plasma glucose (mM) & $5.6 \pm 0.5$ & $9.4 \pm 3.0^{* * *}$ & $9.3 \pm 3.8^{* * *}$ \\
\hline $\operatorname{AlC}(\%)$ & ND & $7.6 \pm 1.2$ & $7.5 \pm 1.5$ \\
\hline$(\mathrm{mmol} / \mathrm{mol})$ & & $60 \pm 13$ & $58 \pm 17$ \\
\hline Systolic BP (mmHg) & $135 \pm 13$ & $136 \pm 13$ & $153 \pm 22^{* * *}$ \\
\hline Diastolic BP (mmHg) & $84 \pm 6$ & $81 \pm 10$ & $84 \pm 10$ \\
\hline Total cholesterol (mM) & $5.52 \pm 0.94$ & $5.04 \pm 1.13$ & $5.17 \pm 1.14$ \\
\hline LDL cholesterol (mM) & $3.31 \pm 0.69$ & $2.92 \pm 0.96$ & $2.58 \pm 0.92^{*}$ \\
\hline HDL cholesterol (mM) & $1.49 \pm 0.32$ & $1.46 \pm 0.38$ & $1.40 \pm 0.29$ \\
\hline Urinary albumin (mg/24h) & ND & $13 \pm 10$ & $2437(371-9000)^{* * *}$ \\
\hline $\mathrm{eGFR}(\mathrm{ml} / \mathrm{min})$ & $69 \pm 13$ & $73 \pm 13$ & $31.7(20.0-45.3)^{* * *, \mathrm{OOO}}$ \\
\hline
\end{tabular}

$\mathrm{ND}=$ not determined. Data are mean $\pm \mathrm{SD}$ or median (minimum-maximum). Significance: ${ }^{*}$ and ${ }^{* * *}, P<0.05$ and $P<0.001$ with respect to healthy volunteers, and ${ }^{\mathrm{OOO}}, P<0.001$ with respect to patients with type 2 diabetes without nephropathy.

fasting plasma glucose $\geq 7.0 \mathrm{mmol} / \mathrm{L}$, a two-hour value in an oral glucose tolerance test $11.1 \mathrm{mmol} / \mathrm{L}$, or random plasma glucose $11.1 \mathrm{mmol} / \mathrm{L}$ in the presence of symptoms, aged $35-$ 75 with a documented duration of diabetes of $\geq 5$ years being eligible. T2DM was diagnosed by lack of criteria for type 1 diabetes. Inclusion criteria for cases were albuminuria $>300 \mathrm{mg} / \mathrm{d}$ and known overt diabetic retinopathy. Retinopathy was requested to be present to ensure that albuminuria is the consequence of diabetic nephropathy rather than a nondiabetic glomerulopathy. A renal biopsy would be the gold standard to discriminate between diabetic nephropathy and a nondiabetic glomerulopathy, but a renal biopsy is rarely taken in patients with T2DM. Several studies have indicated that presence of retinopathy is a good alternative inclusion criterion to discriminate between diabetic nephropathy and nondiabetic glomerulopathy in patients with T2DM with albuminuria [21-23]. Exclusion criteria were chronic renal failure, known causes of renal failure other than diabetes, and non-Caucasian ethnic origin. Cases and controls were matched for gender and diabetes duration. Exclusion criteria for T2DM-DN were microalbuminuria, non-Caucasian ethnic origin, and, in case of use of RAASblocking medication, unknown albuminuria status prior to start of treatment. Estimated GFR (eGFR) was determined by the Chronic Kidney Disease Epidemiology Collaboration equations: for females (for whom [Creatinine $]_{\text {plasma }}$ was $>62 \mu \mathrm{M})$, eGFR $=144 \times\left([\text { Creatinine }]_{\text {plasma }} / 62\right)^{-1.209} \times$ $(0.993)^{\mathrm{Age}}$; and, for males, when [Creatinine $]_{\text {plasma }} \leq$ $80 \mu \mathrm{M}$, eGFR $=141 \times\left([\text { Creatinine }]_{\text {plasma }} / 80\right)^{-0.411} \times$ $(0.993)^{\text {Age }}$, and when [Creatinine $]_{\text {plasma }}>80 \mu \mathrm{M}$, eGFR $=$ $141 \times\left([\text { Creatinine }]_{\text {plasma }} / 80\right)^{-1.209} \times(0.993)^{\text {Age }}[24]$. Glycated haemoglobin (A1C) was determined by a validated ion exchange high pressure chromatography method (Menarini
ARKRAY ADAMS A1C HA-8180 analyser, Menarini Diagnostics, Florence, Italy) [25]. Subject characteristics are given in Table 1.

2.2. Methods. Venous blood samples were collected from all study subjects after overnight fast and plasma samples were prepared immediately and stored at $-80^{\circ} \mathrm{C}$ until analysis; $24 \mathrm{~h}$ urines collections were made and aliquots prepared and stored at $-80^{\circ} \mathrm{C}$ until analysis. Ultrafiltrates were prepared by microspin ultrafiltration (10 kDa cut-off) of plasma and urine $(100 \mu \mathrm{L})$, collecting $c a .50 \mu \mathrm{L}$ ultrafiltrate. Sample processing and storage validation were published previously [5].

Nucleotide markers of glycation and oxidation were determined by stable isotopic dilution analysis LC-MS/MS. GdG, MGdG, CEdG, and 8-OxodG and related stable isotopic $\left[{ }^{13} \mathrm{C}_{10},{ }^{15} \mathrm{~N}_{5}\right]$ substituted standards were prepared as described [5]. For LC-MS/MS, plasma and urine ultrafiltrates $(40 \mu \mathrm{L})$ were spiked with $10 \mu \mathrm{L}$ isotopic standard mixture containing $0.1 \mathrm{nmol}\left[{ }^{13} \mathrm{C}_{10},{ }^{15} \mathrm{~N}_{5}\right] \mathrm{dG}, 1 \mathrm{pmol}\left[{ }^{13} \mathrm{C}_{10},{ }^{15} \mathrm{~N}_{5}\right]$ 8OxodG, 1 pmol $\left[{ }^{13} \mathrm{C}_{10},{ }^{15} \mathrm{~N}_{5}\right]$ MGdG and CEdG, and $1 \mathrm{pmol}$ $\left[{ }^{13} \mathrm{C}_{10},{ }^{15} \mathrm{~N}_{5}\right]$ GdG. LC-MS/MS was performed using an Acquity UPLC-Quattro Premier tandem mass spectrometer with a BEH C18 $1.7 \mu \mathrm{m}$ particle size, $2.1 \times 100 \mathrm{~mm}$ column. The mobile phase $(0.25 \mathrm{~mL} / \mathrm{min})$ was $0.1 \%$ formic acid with a linear gradient of $0-10 \%$ acetonitrile from 2 to $10 \mathrm{~min}$ and isocratic $10 \%$ acetonitrile from 10 to $15 \mathrm{~min}$. After analysis, the column was washed with $50 \%$ acetonitrile, $0.1 \%$ formic acid for $10 \mathrm{~min}$ and thereafter reequilibrated with initial mobile phase for $10 \mathrm{~min}$. The column temperature was varied from $10^{\circ} \mathrm{C}$. For GdG, MGdG, CEdG, and 8-OxodG, limits of detection were $0.8,2.5,2.2$, and $0.7 \mathrm{fmol}$; analytical recoveries were $104,97,98 \%$, and $99 \%$, respectively, and coefficients of variation $2-7 \%[5]$. 
2.3. Statistical Analysis. Data are median (minimum-maximum) or median (lower-upper quartile) values. Significance of differences between means was assessed by Mann-Whitney $U$ test. Bivariate regression was nonparametric (Spearman) and logistic regression was performed of $\mathrm{DN}$ on continuous variables, excluding the recruitment qualifier of urinary albumin, solving for regression coefficient $B$. Statistical analysis was performed by the SPSS software, v21.

\section{Results}

3.1. DNA Glycation Adducts in Plasma and Urine. Biomarkers of nucleotide glycation and oxidation are conveniently determined by assay of glycated and oxidised nucleosides in plasma and urine ultrafiltrates. This was performed with samples from healthy controls and patients with T2DM with and without diabetic nephropathy. Median plasma concentrations of $\mathrm{GdG}, \mathrm{MGdG}, \mathrm{CEdG}$, and 8-OxodG in healthy subjects were $0.07,0.34,0.14$, and $0.08 \mathrm{nM}$, respectively. Plasma GdG was increased 6-fold and plasma 8-OxodG increased 2-fold in patients with T2DM. Median urinary excretion rates of GdG, MGdG, CEdG, and 8-OxodG in healthy subjects were $0.23,2.63,0.90$, and $0.90 \mathrm{nmol} / 24 \mathrm{~h}$, respectively. In patients with T2DM, urinary GdG, MGdG, CEdG, and 8-OxodG were increased 10-fold, 2-fold, 2-fold, and 28-fold, respectively, Figures 1(b)-1(i).

We also assessed the effect of diabetic nephropathy (DN) in patients with T2DM on plasma and urinary nucleotide glycation and oxidation damage markers. In plasma, median 8-OxodG concentration was increased $30 \%$ in $\mathrm{DN}$ and in urine GdG was increased 24\% and CEdG increased 60\% in diabetic nephropathy, Figures 1(j)-1(l).

3.2. Correlation Analysis. Bivariate correlation analysis of clinical chemistry variables of renal function with nucleoside glycation and oxidation analytes in patients with T2DM is given in Table 2. As expected, there were a strong negative correlation of plasma creatinine with eGFR $(r=-0.95, P<$ 0.001 ), a strong positive correction of plasma creatinine with urinary albumin excretion UAE $(r=0.61, P<0.001)$, and a strong negative correction of eGFR with UAE $(r=-0.55$, $P<0.001)$. There were also positive correlations of urinary excretions of GdG and CEdG with UAE ( $r=0.38$ and 0.42 , resp., $P<0.01$ ) and a positive correlation of plasma 8 OxodG with plasma creatinine $(r=0.38, P<0.01)$. For correlation between nucleotide glycation and oxidation analytes, in plasma there were a strong positive correlation of MGdG with CEdG $(r=0.46, P<0.001)$ and also positive correlation of MGdG with 8-OxodG $(r=0.45, P<0.01)$ and GdG with CEdG $(r=0.47, P<0.01)$. For urinary excretion, there was a positive correlation of MGdG excretion with 8OxodG excretion $(r=0.37, P<0.01)$. There was also a positive correlation of plasma MGdG with urinary MGdG $(r=0.41, P<0.01)$ but there were no similar correlations of plasma and urinary levels of other nucleotide glycation and oxidation analytes. There was no correlation of clinical chemistry variables of glycemic control (fasting plasma glucose, A1C), total cholesterol, LDL cholesterol, HDL cholesterol, or systolic and diastolic blood pressure with nucleotide glycation and oxidation markers.

In a multiple logistic regression analysis of diabetic nephropathy on clinical and clinical chemistry variables, the following variables were included: clinical variables previously associated with diabetic nephropathy (age, gender, A1C, systolic and diastolic blood pressure, and total cholesterol) $[21,26]$, related markers of metabolic control (fasting plasma glucose, LDL, and HDL), other factors linked to increased glyoxal and methylglyoxal (duration of diabetes, BMI) [1, 22], and markers of DNA glycation and oxidation measured noninvasively (urinary excretions of GdG, MGdG, CEdG, and 8-OxodG). Plasma creatinine, eGFR, and UAE were excluded from the model as established biomarkers of diabetic nephropathy. Forward stepwise selection of variables gave a multiple logistic regression model linking diabetic nephropathy to systolic blood pressure $(B=0.05 \pm 0.02$, exponent $1.05 ; P=0.009)$ and urinary excretion of $\mathrm{CEdG}$ $(B=1.00 \pm 0.41$, exponent 2.70; $P=0.016, n=56)$. This is consistent with the increased systolic blood pressure found in patients with diabetic nephropathy and the positive correlation of urinary CEdG with UAE, Tables 1 and 2.

\section{Discussion}

Herein we found, for the first time, increased plasma levels and urinary excretion of DNA glycation adducts in patients with T2DM and a link to diabetic nephropathy. Hence, measurement of DNA glycation adducts and nucleosides in body fluids may be valuable biomarkers of quantitative and functional important DNA damage in vivo. Glyoxal is formed in physiological systems by lipid peroxidation and degradation of glycated proteins and monosaccharides. Methylglyoxal is formed mainly by degradation of triosephosphates and also by ketone body metabolism and threonine catabolism [23]. Both dicarbonyls are metabolised by the glutathionedependent Glo1 [6] recently linked mechanistically to the development of diabetic nephropathy [14]. Glycation of DNA by glyoxal and methylglyoxal has been linked to DNA strand breaks and mutagenesis [27, 28], and cellular dicarbonyl concentrations increase in oxidative stress [29]. Formation of MGdG and CEdG by glycation of DNA with endogenous methylglyoxal may explain the previously reported enhancement of DNA modification by glucose metabolites under conditions of glutathione depletion [30], increased DNA unwinding, and single-strand breaks of DNA in vascular endothelial cell in hyperglycemia in vitro [31] and increased single-strand breaks of DNA of patients with diabetes [32].

In previous studies we found levels of GdG and CEdG residues in peripheral lymphocyte DNA of healthy people to be similar to those of 8-OxodG, and the DNA content of MGdG exceeded that of the widely studied 8-OxodG [5]. Nucleotide AGE content of DNA was also markedly higher than that of other physiological aldehydes-such as 4-hydroxynonenal and malondialdehyde [33]. Modification of DNA by physiological dicarbonyls therefore gives rise to quantitatively important steady-state levels of deoxyguanosine-derived adducts in cellular DNA in vivo. 


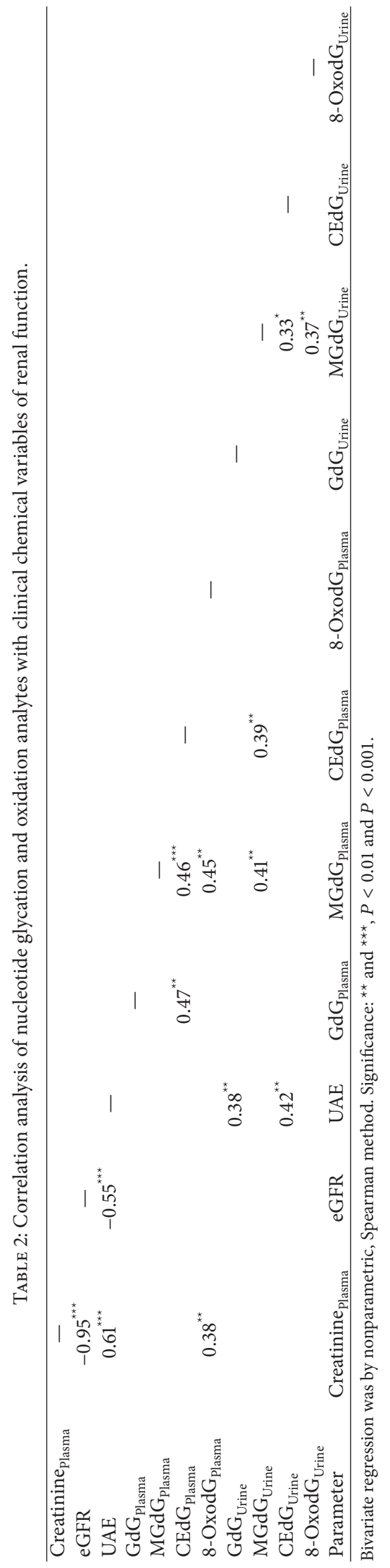


In patients with $\mathrm{T} 2 \mathrm{DM}$, there were particularly large increases in glyoxal-derived imidazopurinone GdG in plasma and urine and 8-OxodG in urine. We cannot discriminate between the contributions to glycated and oxidised nucleosides formed by repair of glycated and oxidised DNA and formed by direct glycation and oxidation of deoxyguanosine. Formation of GdG in patients with T2DM appears to be particularly favoured. For methylglyoxal-derived metabolites, CEdG is more stable chemically than MGdG and may be a more robust biomarker when released from cells [5]. LC-MS/MS analysis of urinary excretion of CEdG in streptozotocin-induced diabetic rats indicated a 4 -fold increase in CEdG excretion in diabetes with respect to normal healthy control rats [34]. Increased formation of glyoxal and methylglyoxal and oxidative stress in hyperglycemia associated with diabetes has been linked to microvascular complications-including diabetic nephropathy $[1,35]$. Related damage to proteins [36] and herein DNA provides potential markers of nephropathy development.

We assessed the link of urinary excretion of nucleoside glycation and oxidation adducts as potential noninvasive biomarkers of diabetic nephropathy. In multiple logistic regression analysis, urinary CEdG emerged as a positive correlate for diabetic nephropathy. Urinary CEdG also correlated positively with UAE and hence is linked to an established biomarker of diabetic nephropathy. It also likely reflects increased exposure to methylglyoxal. CEdG has higher chemical stability than MGdG and higher biological stability than methylglyoxal in cells and physiological fluids which may explain its greater diagnostic value than these related metabolites $[5,22]$.

Plasma and urinary 8-OxodG were also increased in patients with T2DM. Urinary oxidation adducts of RNA rather than DNA were associated with mortality in a prospective study of patient with T2DM [37]. Increased urinary 8OxodG in patients with T2DM was found previously but the technique used, liquid chromatography with electrochemical detection, appears to have overestimated urinary levels of 8 -OxodG by $c a$. 10 -fold $[11,12]$. The greater sensitivity and specificity for analyte detection provided by multiple reaction monitoring detection in LC-MS/MS accounts for this. The two major analytical approaches that have been used for the measurement of urinary 8-OxodG prior to application of LC-MS/MS were HPLC combined with electrochemical detection and immunoassay. Approaches other than LCMS/MS have overestimated 8-OxodG [38-40]. Estimates of urinary 8-OxodG herein are similar to independent estimates by stable isotopic dilution analysis LC-MS/MS for healthy controls [41] and patients with T2DM [42]. Similar improvements have been made in the detection of CEdG by LC-MS/MS. CEdG was determined in urine of normal healthy human subjects previously by immunoassay with estimates in the range of $3.4-344 \mathrm{pmol} / \mathrm{mg}$ creatinine and median of $c a .30 \mathrm{pmol} / \mathrm{mg}$ creatinine [43]. Estimation herein by LC-MS/MS gave median (minimum-maximum) values of 0.55 (0.17-1.58) $\mathrm{pmol} / \mathrm{mg}$ creatinine, suggesting that immunoassay procedures overestimated urinary CEdG by ca. 50-fold, as was found for similar ELISA and LC-MS/MS measurement of 8-OxodG [39]. Overestimation is likely caused by interference due to imperfect epitope specificity of the monoclonal antibody used and formation of CEdG from MGdG and other sample components during the high $\mathrm{pH}$ of preanalytic sample processing.

A weakness of this study was lack of females in the healthy control study group but there was no indication in the T2DM group that DNA damage marker levels were linked to gender.

\section{Conclusion}

From the quantitative amount and link to functional endpoints, GdG, MGdG, and CEdG adducts are of likely pathogenic and diagnostic significance. Recent further evidence linking dicarbonyl glycation with development of DN [14] suggests that DNA dicarbonyl adducts may emerge as biomarkers of development of DN. This study suggests that dicarbonyl adducts are not surrogate measures of metabolic control and some are linked to DN.

\author{
Abbreviations \\ A1C: $\quad$ Glycated haemoglobin \\ AGE: $\quad$ Advanced glycation endproduct \\ CEdG and N2-(1,R/S-Carboxyethyl)- \\ $\mathrm{CEdG}_{\mathrm{A}} / \mathrm{CEdG}_{\mathrm{B}}$ : deoxyguanosine and $\mathrm{R} / \mathrm{S}$ \\ epimers \\ dG: Deoxyguanosine \\ GdG: $\quad 3-\left(2^{\prime}\right.$-Deoxyribosyl)-6,7-dihydro-6,7- \\ eGFR: $\quad$ Estimated glomerular filtration rate \\ Glo1: $\quad$ Glyoxalase 1 \\ 8-OxodG: $\quad$ 7,8-Dihydro-8-oxo-2 ${ }^{\prime}$-deoxyguanosine \\ 4HNE: 4-Hydroxynonenal \\ MGdG: $\quad$ 3-(2'-Deoxyribosyl)-6,7-dihydro-6,7- \\ dihydroxy-6/7-methylimidazo-[2,3- \\ b]purine-9(8)one \\ LC-MS/MS: Liquid chromatography with tandem \\ mass spectrometric detection \\ UAE: $\quad$ Urinary albumin excretion \\ DN: Diabetic nephropathy.
}

\section{Conflict of Interests}

The authors declare that there is no conflict of interests regarding the publication of this paper.

\section{Authors' Contribution}

Sahar Waris researched the data, Brigitte M. WinklhoferRoob, Johannes M. Roob, Sebastian Fuchs, and Harald Sourij performed subject recruitment and sample collection, Brigitte M. Winklhofer-Roob also edited the paper, and Naila Rabbani and Paul J. Thornalley researched the data and wrote the paper. 


\section{Acknowledgments}

Sahar Waris thanks the Commonwealth Scholarship Commission for a split-site Ph.D. studentship and was on secondment from Department of Biochemistry, Faculty of Medicine, Jawaharlal Nehru Medical College, Aligarh Muslim University, Aligarh PIN 202002, India. The project was also funded by the Commission of the European Communities, 6th Framework Programme Priority 1, Life Sciences, Genomics and Biotechnology for Health, LSHM-CT-2005-018733, PREDICTIONS (Prevention of Diabetic Complications), and 5th Framework Programme, Key Action 1, Food, Nutrition and Health, QLK1-CT-1999-00830, VITAGE (Vitamin A, Vitamin $\mathrm{E}$ and Carotenoid Status and Metabolism during Ageing: Functional and Nutritional Consequences). The authors thank Astrid Knopf and Sandra Wuga, Human Nutrition AND Metabolism Research and Training Center, Institute of Molecular Biosciences, Karl Franzens University of Graz, Austria, for excellent technical assistance.

\section{References}

[1] A. C. McLellan, P. J. Thornalley, J. Benn, and P. H. Sonksen, "Glyoxalase system in clinical diabetes mellitus and correlation with diabetic complications," Clinical Science, vol. 87, no. 1, pp. 21-29, 1994.

[2] N. Rabbani and P. J. Thornalley, "Dicarbonyls (glyoxal, methylglyoxal, and 3-deoxyglucosone)," in Uremic Toxins, pp. 177-192, John Wiley \& Sons, 2012.

[3] J. W. Baynes, "Role of oxidative stress in development of complications in diabetes," Diabetes, vol. 40, no. 4, pp. 405-412, 1991.

[4] P. Dandona, K. Thusu, S. Cook et al., "Oxidative damage to DNA in diabetes mellitus," The Lancet, vol. 347, no. 8999, pp. 444-445, 1996.

[5] P. J. Thornalley, S. Waris, T. Fleming et al., "Imidazopurinones are markers of physiological genomic damage linked to DNA instability and glyoxalase 1-associated tumour multidrug resistance," Nucleic Acids Research, vol. 38, no. 16, Article ID gkq306, pp. 5432-5442, 2010.

[6] M. Xue, N. Rabbani, and P. J. Thornalley, "Glyoxalase in ageing," Seminars in Cell and Developmental Biology, vol. 22, no. 3, pp. 293-301, 2011.

[7] A. Bierhaus, T. Fleming, S. Stoyanov et al., "Methylglyoxal modification of $\mathrm{Na}_{v} 1.8$ facilitates nociceptive neuron firing and causes hyperalgesia in diabetic neuropathy," Nature Medicine, vol. 18, no. 6, pp. 926-933, 2012.

[8] P. Palsamy and S. Subramanian, "Resveratrol protects diabetic kidney by attenuating hyperglycemia-mediated oxidative stress and renal inflammatory cytokines via Nrf2-Keap1 signaling," Biochimica et Biophysica Acta, vol. 1812, no. 7, pp. 719-731, 2011.

[9] M. T. Barati, M. L. Merchant, A. B. Kain, A. W. Jevans, K. R. McLeish, and J. B. Klein, "Proteomic analysis defines altered cellular redox pathways and advanced glycation end-product metabolism in glomeruli of $\mathrm{db} / \mathrm{db}$ diabetic mice," American Journal of Physiology: Renal Physiology, vol. 293, no. 4, pp. F1157F1165, 2007.

[10] N. Karachalias, R. Babaei-Jadidi, N. Rabbani, and P. J. Thornalley, "Increased protein damage in renal glomeruli, retina, nerve, plasma and urine and its prevention by thiamine and benfotiamine therapy in a rat model of diabetes," Diabetologia, vol. 53, no. 7, pp. 1506-1516, 2010.
[11] Y. Hinokio, S. Suzuki, M. Hirai, M. Chiba, A. Hirai, and T. Toyota, "Oxidative DNA damage in diabetes mellitus: its association with diabetic complications," Diabetologia, vol. 42, no. 8, pp. 995-998, 1999.

[12] Y. Hinokio, S. Suzuki, M. Hirai, C. Suzuki, M. Suzuki, and T. Toyota, "Urinary excretion of 8-oxo-7, 8-dihydro-2'-deoxyguanosine as a predictor of the development of diabetic nephropathy," Diabetologia, vol. 45, no. 6, pp. 877-882, 2002.

[13] W. Seidel and M. Pischetsrieder, "DNA-glycation leads to depurination by the loss of N2-carboxyethylguanine in vitro," Cellular and Molecular Biology, vol. 44, no. 7, pp. 1165-1170, 1998.

[14] F. Giacco, X. Du, V. D. D’Agati et al., "Knockdown of glyoxalase 1 mimics diabetic nephropathy in nondiabetic mice," Diabetes, vol. 63, no. 1, pp. 291-299, 2014.

[15] N. Rabbani and P. J. Thornalley, "The critical role of methylglyoxal and glyoxalase 1 in diabetic nephropathy," Diabetes, vol. 63, no. 1, pp. 50-52, 2014.

[16] C. E. Vaca, J. L. Fang, M. Conradi, and S.-M. Hou, "Development of a 32P-postlabelling method for the analysis of $2^{\prime}$-deoxyguanosine- $3^{\prime}$-monophosphate and DNA adducts of methylglyoxal," Carcinogenesis, vol. 15, no. 9, pp. 1887-1894, 1994.

[17] H. Li, S. Nakamura, S. Miyazaki et al., " $N{ }^{2}$-carboxyethyl$2^{\prime}$-deoxyguanosine, a DNA glycation marker, in kidneys and aortas of diabetic and uremic patients," Kidney International, vol. 69, no. 2, pp. 388-392, 2006.

[18] M. S. Cooke, R. Olinski, S. Loft, and European Standards Committee on Urinary Lesion, "Measurement and meaning of oxidatively modified DNA lesions in urine," Cancer Epidemiol Biomarkers Prevention, vol. 17, no. 1, pp. 3-14, 2008.

[19] K. Rossing, H. Mischak, M. Dakna et al., "Urinary proteomics in diabetes and CKD," Journal of the American Society of Nephrology, vol. 19, no. 7, pp. 1283-1290, 2008.

[20] E. Rock, B. M. Winklhofer-Roob, J. Ribalta et al., "Vitamin A, vitamin $\mathrm{E}$ and carotenoid status and metabolism during ageing: functional and nutritional consequences (Vitage Project)," Nutrition, Metabolism and Cardiovascular Diseases, vol. 11, no. 4, supplement, pp. 70-73, 2001.

[21] American-Diabetes-Association, "Standards of Medical Care in Diabetes-2014," Diabetes Care, vol. 37, supplement 1, pp. S14S80, 2013.

[22] N. Rabbani and P. J. Thornalley, "Dicarbonyl stress in cell and tissue dysfunction contributing to ageing and disease," Biochemical and Biophysical Research Communications, vol. 458, no. 2, pp. 221-226, 2015.

[23] P. J. Thornalley, "Dicarbonyl intermediates in the Maillard reaction," Annals of the New York Academy of Sciences, vol. 1043, pp. 111-117, 2005.

[24] F. Iliadis, T. Didangelos, A. Ntemka et al., "Glomerular filtration rate estimation in patients with type 2 diabetes: creatinine- or cystatin C-based equations?” Diabetologia, vol. 54, no. 12, pp. 2987-2994, 2011.

[25] E. Urrechaga, E. Bereciartua, E. Crespo, and C. Izcara, "Evaluation of AdamsTM AlC Menarini HA-8180 HPLC analyser for HbAlc determination," Clinical Chemistry and Laboratory Medicine, vol. 49, p. S406, 2011.

[26] M.-A. Gall, P. Hougaard, K. Borch-Johnsen, and H.-H. Parving, "Risk factors for development of incipient and overt diabetic nephropathy in patients with non-insulin dependent diabetes mellitus: prospective, observational study," British Medical Journal, vol. 314, no. 7083, pp. 783-788, 1997. 
[27] N. Murata-Kamiya, H. Kamiya, H. Kaji, and H. Kasai, "Glyoxal, a major product of DNA oxidation, induces mutations at G:C sites on a shuttle vector plasmid replicated in mammalian cells," Nucleic Acids Research, vol. 25, no. 10, pp. 1897-1902, 1997.

[28] N. Murata-Kamiya, H. Kamiya, H. Kaji, and H. Kasai, "Methylglyoxal induces $\mathrm{G}: \mathrm{C}$ to $\mathrm{C}: \mathrm{G}$ and $\mathrm{G}: \mathrm{C}$ to $\mathrm{T}: \mathrm{A}$ transversions in the supF gene on a shuttle vector plasmid replicated in mammalian cells," Mutation Research, vol. 468, no. 2, pp. 173-182, 2000.

[29] E. A. Abordo, H. S. Minhas, and P. J. Thornalley, "Accumulation of $\alpha$-oxoaldehydes during oxidative stress: a role in cytotoxicity," Biochemical Pharmacology, vol. 58, no. 4, pp. 641-648, 1999.

[30] T. K. Shires, J. Tresnak, M. Kaminsky, S. L. Herzog, and B. TrucPham, "DNA modification in vivo by derivatives of glucose: enhancement by glutathione depletion," The FASEB Journal, vol. 4, no. 15, pp. 3340-3346, 1990.

[31] M. Lorenzi, D. F. Montisano, S. Toledo, and A. Barrieux, "High glucose induces DNA damage in cultured human endothelial cells," The Journal of Clinical Investigation, vol. 77, no. 1, pp. 322$325,1986$.

[32] M. Lorenzi, D. F. Montisano, S. Toledo, and H.-C. H. Wong, "Increased single strand breaks in DNA of lymphocytes from diabetic subjects," Journal of Clinical Investigation, vol. 79, no. 2, pp. 653-656, 1987.

[33] R. de Bont and N. van Larebeke, "Endogenous DNA damage in humans: a review of quantitative data," Mutagenesis, vol. 19, no. 3, pp. 169-185, 2004.

[34] T. Synold, B. Xi, G. E. Wuenschell et al., "Advanced glycation end products of DNA: quantification of $N$ 2-(1-carboxyethyl)-2Ideoxyguanosine in biological samples by liquid chromatography electrospray ionization tandem mass spectrometry," Chemical Research in Toxicology, vol. 21, no. 11, pp. 2148-2155, 2008.

[35] M. Brownlee, "Biochemistry and molecular cell biology of diabetic complications," Nature, vol. 414, no. 6865, pp. 813-820, 2001.

[36] N. Rabbani, A. Adaikalakoteswari, K. Rossing et al., "Effect of Irbesartan treatment on plasma and urinary markers of protein damage in patients with type 2 diabetes and microalbuminuria," Amino Acids, vol. 42, no. 5, pp. 1627-1639, 2012.

[37] K. Broedbaek, V. Siersma, T. Henriksen et al., "Association between urinary markers of nucleic acid oxidation and mortality in type 2 diabetes: a population-based cohort study," Diabetes Care, vol. 36, no. 3, pp. 669-676, 2013.

[38] K. Broedbaek, A. Weimann, E. S. Stovgaard, and H. E. Poulsen, "Urinary 8-oxo-7,8-dihydro-2I-deoxyguanosine as a biomarker in type 2 diabetes," Free Radical Biology and Medicine, vol. 51, no. 8, pp. 1473-1479, 2011.

[39] M. S. Cooke, R. Singh, G. K. Hall et al., "Evaluation of enzymelinked immunosorbent assay and liquid chromatographytandem mass spectrometry methodology for the analysis of 8oxo-7,8-dihydro-2/-deoxyguanosine in saliva and urine," Free Radical Biology and Medicine, vol. 41, no. 12, pp. 1829-1836, 2006.

[40] L. Barregard, P. Møller, T. Henriksen et al., "Human and methodological sources of variability in the measurement of urinary 8-oxo-7,8-dihydro-2' -deoxyguanosine," Antioxidants and Redox Signaling, vol. 18, no. 18, pp. 2377-2391, 2013.

[41] K. Broedbaek, R. Ribel-Madsen, T. Henriksen et al., "Genetic and environmental influences on oxidative damage assessed in elderly Danish twins," Free Radical Biology and Medicine, vol. 50, no. 11, pp. 1488-1491, 2011.
[42] K. Broedbaek, T. Henriksen, A. Weimann et al., "Long-term effects of Irbesartan treatment and smoking on nucleic acid oxidation in patients with type 2 diabetes and microalbuminuria: an irbesartan in patients with type 2 diabetes and microalbuminuria (IRMA 2) substudy," Diabetes Care, vol. 34, no. 5, pp. 1192-1198, 2011.

[43] M. Schneider, G. Thoss, C. Hübner-Parajsz, R. Kientsch-Engel, P. Stahl, and M. Pischetsrieder, "Determination of glycated nucleobases in human urine by a new monoclonal antibody specific for $N^{2}$-carboxyethyl-2/-deoxyguanosine," Chemical Research in Toxicology, vol. 17, no. 10, pp. 1385-1390, 2004. 


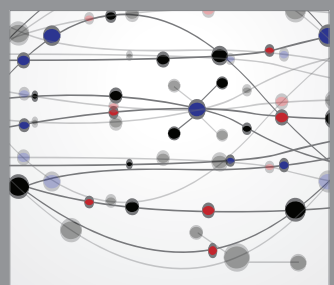

The Scientific World Journal
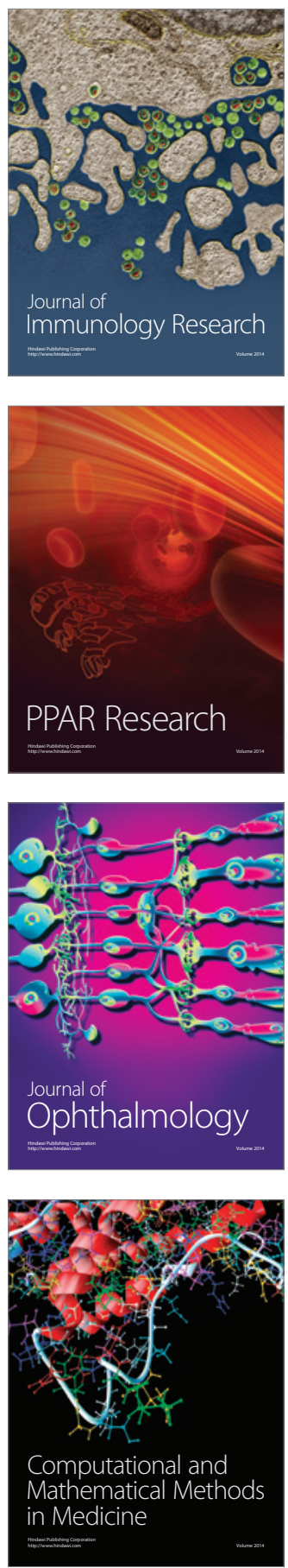

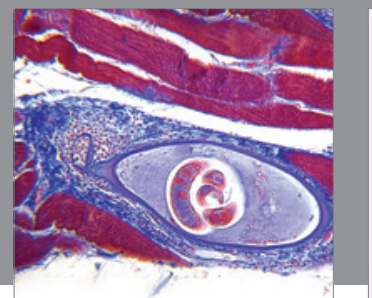

Gastroenterology

Research and Practice
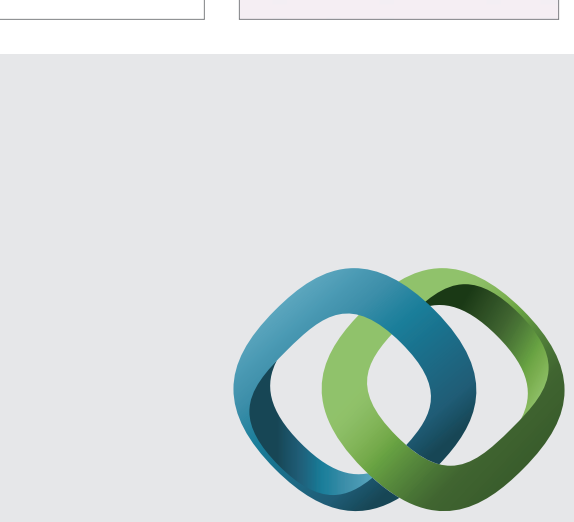

\section{Hindawi}

Submit your manuscripts at

http://www.hindawi.com
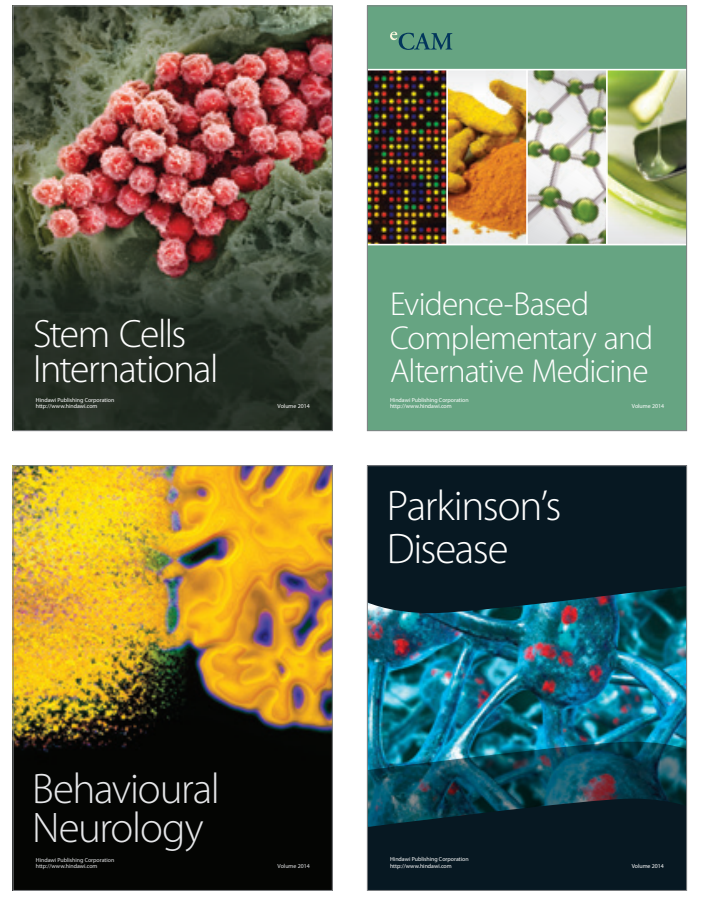
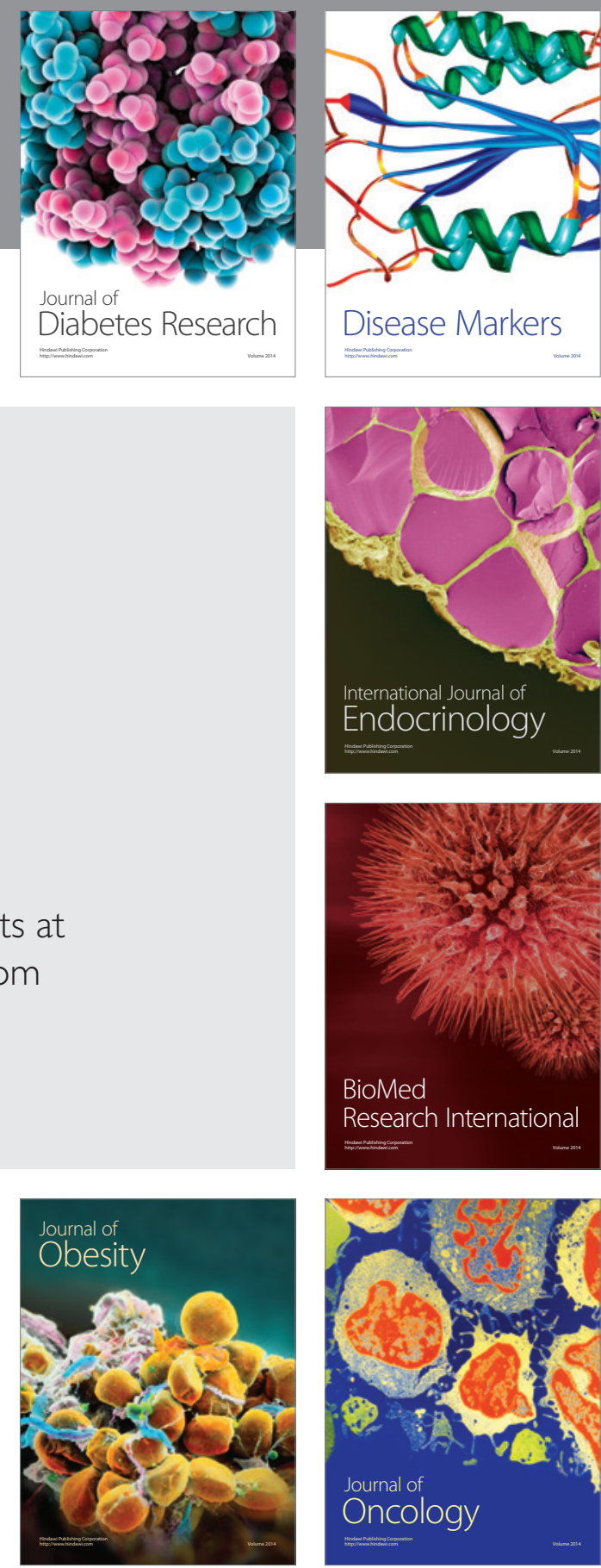

Disease Markers
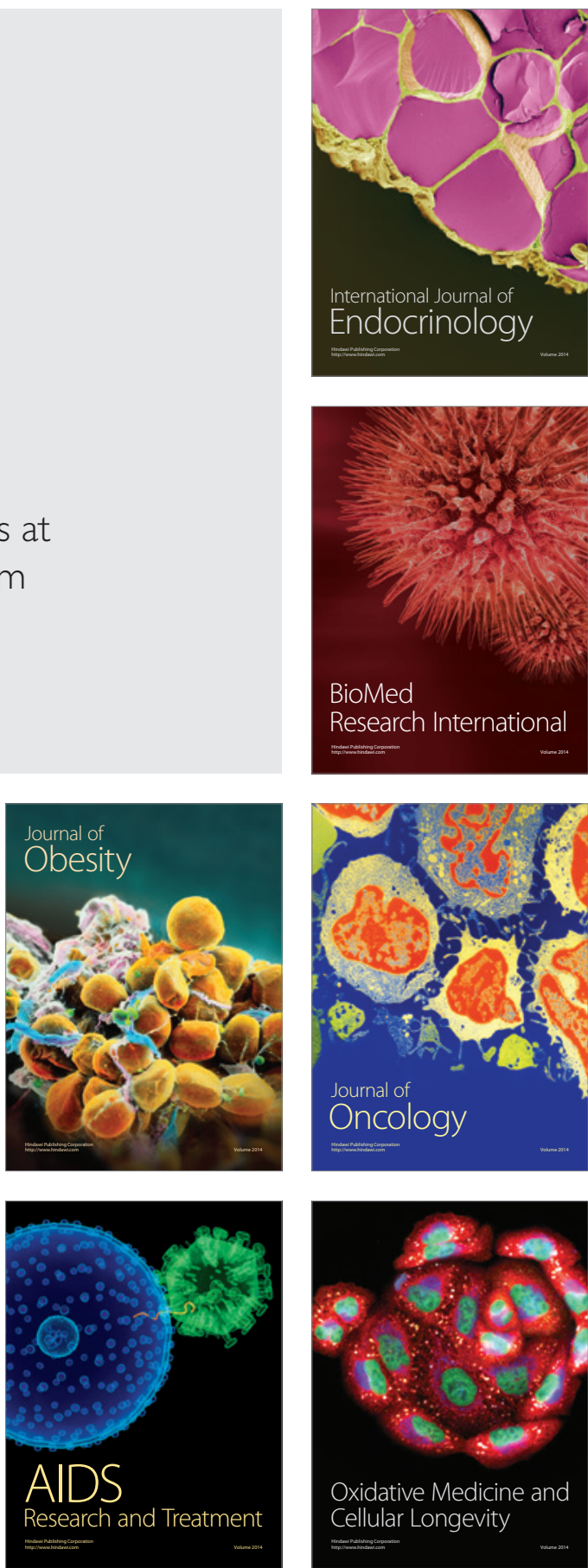